Received: 26 April 2017

Accepted: 28 June 2017

Published online: 2 August 2017

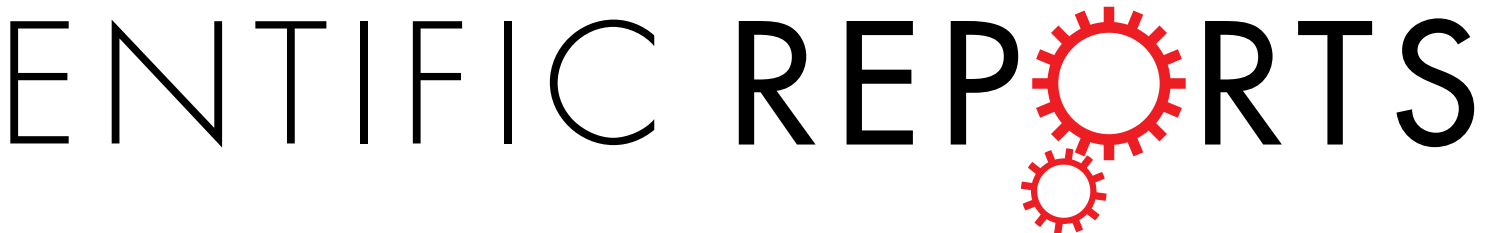

\section{The association between structure- function relationships and cognitive impairment in elderly glaucoma patients}

\author{
Megumi Honjo ${ }^{1,2}$, Jiro Numaga ${ }^{2}$, Tadashi Hara ${ }^{3} \&$ Ryo Asaoka ${ }^{1}$
}

Accurate measurement of visual field (VF) is important in accessing glaucoma, however this may not be achieved in patients with dementia or mild cognitive impairment $(\mathrm{Cl})$. We investigated the association between $\mathrm{Cl}$ and structure-function relationships in elderly glaucoma patients. The study included 94 eyes of 51 glaucoma patients aged $\geq 75$ years with no diagnoses of dementia. $\mathrm{Cl}$ was assessed using the Mini Mental State Examination (MMSE). Using the leave-one-out cross-validation, the mean deviation (MD) of the Humphrey 30-2 VF was predicted from measurements of optical coherence tomography, and the relationship between the squared prediction error and the MMSE score, together with age, fixation loss (FL), false positive (FP), and false negative (FN) percentages that were analyzed using the linear mixed model. A high prevalence of $\mathrm{MCl}$ or dementia was observed in the elderly population. The squared prediction error value of the MD was $17.0 \pm 21.1$ (mean \pm standard deviation). The squared prediction error increased with decreasing MMSE total score, but age, FL, FP, and FN were not related. Careful consideration is needed when interpreting the VF results of these patients, because VF can be over- or underestimated, as suggested by the decreased structure-function relationships.

Glaucoma is the second leading cause of blindness in the world ${ }^{1}$. It is characterized by progressive visual field (VF) damage, which affects the quality of life ${ }^{2}$. Glaucoma was present in 60.5 million people worldwide in 2010, but this number is estimated to rise to 79.6 million by 2020 , because of the aging population and because the prevalence of ocular diseases increases with increasing age ${ }^{3-6}$. The aged population faces other problems, such as cognitive impairment and dementia, which affect their independent and active lives, and may eventually be fatal ${ }^{7}$. Dementia is a decline of cognitive function severe enough to interfere with social function, and is not associated with changes in consciousness. Alzheimer's disease $(\mathrm{AD})$ is the most common form of dementia, and the prevalence of dementia has been reported to increase rapidly with advancing age ${ }^{8}$. Dementia reportedly affects 36 million people globally ${ }^{9}$. The true prevalence of missed and delayed diagnoses of dementia is unknown, but is likely high ${ }^{10}$. Additionally, mild cognitive impairment (MCI), which is a risk factor for the development of dementia but not sufficient for a diagnosis of dementia ${ }^{11}$, has become a major health problem among older patients because of its high prevalence in the aged population. Approximately $15-42 \%$ of patients aged $\geq 65$ years are estimated to have MCI, and approximately $5-15 \%$ of these patients progress to dementia annually ${ }^{12}$, suggesting that high rates of diagnosed/undiagnosed dementia or MCI occur in glaucoma patients.

Reliable VF results are required to achieve an accurate diagnosis of glaucoma as early as possible ${ }^{13,14}$. However, this may not be achieved in patients with dementia or MCI. Fixation losses (FLs), false positives (FPs), and false negatives (FNs) can be reliably measured using the Humphrey Field Analyzer (HFA; Carl Zeiss Meditec, Dublin, CA, USA). These indices are important clinical markers. Elevated FLs can mask early scotoma ${ }^{15,16}$, increased FP errors indicate 'trigger-happy' patients, and high FNs may suggest patient inattention or fatigue during the VF examination ${ }^{17-19}$. Previous studies have suggested the usefulness of these indices ${ }^{20,21}$, but more recent studies have reported their limitations in reliably estimating the $\mathrm{VF}^{16,22-24}$.

Recent developments in optical coherence tomography (OCT) have enabled evaluation of the circumpapillary retinal nerve fiber layer (cpRNFL) and the macular ganglion cell complex (GCC) thicknesses ${ }^{25-33}$. 
Previous studies have reported that these structural measurements are related to the $\mathrm{VF}^{28,29,34-41}$. However, these structure-function relationships can be affected by other factors. For example, we previously reported that eye movement, as measured by gaze tracking using the HFA, resulted in improved characterization of structure-function relationships ${ }^{42}$. We recently reported significant correlations between structure-function relationships in elderly glaucoma patients when reliable perimetry was performed ${ }^{43}$. However, VF examinations are often unreliable in elderly patients ${ }^{44}$, and cognitive status in elderly patients could be related to the reliability of the VF measurements.

The Mini-Mental State Examination (MMSE) is a widely used cognitive test that is useful for examining patients with an increased risk of dementia ${ }^{45}$. In the present prospective study, cognitive impairment was assessed using the MMSE in elderly glaucoma patients who did not have a diagnosis of dementia. The objectives of this study were to confirm the prevalence of cognitive impairment in elderly glaucoma patients, to identify possible relationships between cognitive decline and reliable measurements of the VF in these patients, and to determine whether cognitive impairment was related to structure-function relationships in the same patients.

\section{Methods}

Subjects. This prospective, cross-sectional study was conducted at the Tokyo Metropolitan Geriatric Hospital and the Hara Eye Clinic. Only patients $\geq 75$ years of age with no history of dementia were recruited. The Institutional Review Board and Ethics Committee of the institute approved the study, and the study protocol adhered to the tenets of the Declaration of Helsinki. Written informed consent for both study participation and publication of ocular examination images was obtained from each participating patient.

The study initially included 104 eyes of 54 OAG glaucoma patients. All patients visited the glaucoma clinic from July 2015 to November 2015. They fulfilled the following criteria: (1) glaucoma was the only disease causing VF damage; (2) axial length was between $26.0 \mathrm{~mm}$ and $21.0 \mathrm{~mm}$; (3) patients underwent at least three VF tests, prior to the current study (to mitigate learning effects); and (4) logMAR visual acuity $\leq 0.5^{46}$. All VFs were measured using the HFA (30-2 Swedish Interactive Threshold Algorithm, standard program) and those with unreliable VFs defined as $\mathrm{FL}>33 \%, \mathrm{FP}>33 \%$, or $\mathrm{FN}>33 \%$ were excluded ${ }^{47}$. Patients with successful cataract surgery and patients with clinically insignificant cataract were included in the study. After providing written informed consent, all patients underwent an ocular examination including an autorefractometry examination, measurement of best-corrected visual acuity, a slit-lamp examination, measurement of the axial length using the IOL Master (Carl Zeiss Meditec), measurement of the intraocular pressure, dilated fundoscopy, VF measurement, MMSE, and a spectral domain (SD)-OCT examination using a RS-3000 Advance OCT, software version 1.4.2.1 (NIDEK, Gamagori, Japan). All examinations were conducted within 1 month of the VF measurements.

Assessment of cognitive function. Cognitive function was assessed using the MMSE, which was developed by Folstein et al. in 1975 and is widely used as a brief screening test for dementia and as a measure of global cognitive function ${ }^{48}$. It focuses on five downstream items: orientation, memory, concentration, language, and design capacity. It includes 11 questions, and was performed by an experienced examiner ${ }^{49}$.

The MMSE total score ranged from 0-30, with lower scores indicating poorer cognitive ability. Patients with a score of $\leq 23$ points were classified as having cognitive impairment ${ }^{50,51}$. Using the MMSE total score, any score $\geq 28$ points indicated normal cognition, and scores $<28$ points indicated MCI (24-27 points) with moderate (10-23 points) or severe ( $\leq 9$ points) cognitive impairment. A score of $\leq 9$ points was considered to be almost diagnostic of dementia ${ }^{50-52}$.

OCT measurements. OCT examinations were performed using an RS-3000 Advance SD-OCT as previously described $^{53,54}$. For cpRNFL imaging, raster scanning over a $6 \times 6 \mathrm{~mm}^{2}$ area centered on the optic disc center was performed at a scan density of $512 \mathrm{~A}$-scans (horizontal) $\times 128 \mathrm{~B}$-scans (vertical). For wide area three-dimensional imaging of the macula, raster scanning was performed over a $30^{\circ} \times 30^{\circ}$ area (equivalent to a $9 \times 9 \mathrm{~mm}^{2}$ area in the Gullstrand model eye). The cpRNFL/GCC thickness was measured using the built-in software. An experienced examiner $(\mathrm{MH})$ confirmed the validity of the image segmentation, and any images with motion artifacts or incorrect segmentation were excluded from analyses. Only images with a signal strength index $>50$ were included in analyses.

Statistical analysis. To determine how MMSE total score, FL, FP, and FN affected the degree of structure-function relationships, a linear mixed model was constructed between the average cpRNFL thickness and the average GCC thickness against the mean deviation (MD). A linear mixed model was applied to a nested dataset in the current study, and patients were treated as a 'random effect' because both eyes were included in analyses. Then, the prediction error was calculated using the leave-one-out cross-validation method, whereby the data from one or both eyes of a single patient were used for validation, and the data from the remaining subjects were used for training. This procedure was then repeated until each OAG patient in the original sample was used once as validation data. Thus, for each individual, only the data from all other subjects were used in the prediction. Finally, using the variables of age, FL, FP, FN, and MMSE total score, the optimal linear mixed model for the raw prediction error value, the squared prediction error, and the prediction error value (see Fig. 1) were

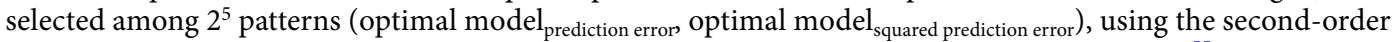
bias-corrected Akaike information criterion index which is a correction for finite sample sizes ${ }^{55}$. All statistical analyses were performed using the statistical programming language ' $R$ ' version 3.2.3 (Foundation for Statistical Computing, Vienna, Austria).

Data Availability. All data generated or analysed during this study are included in this published article. 
Right eye

cpRNFL
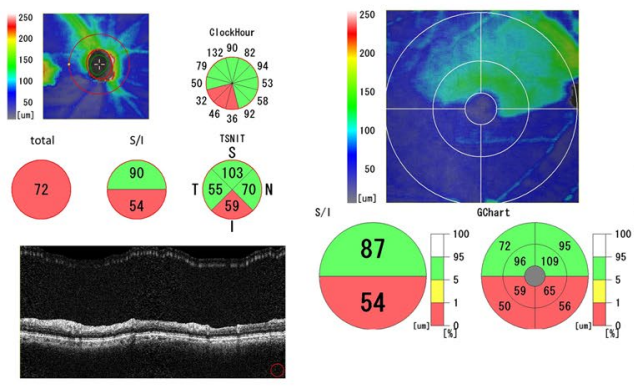

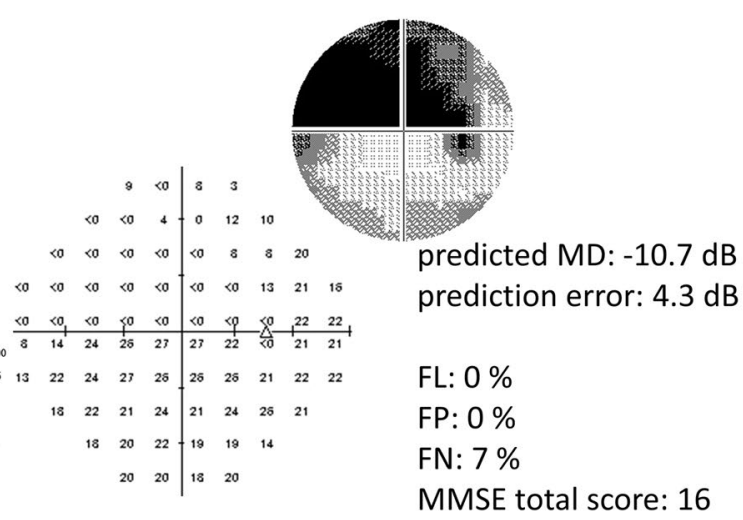

actual $\mathrm{MD}=-15.0 \mathrm{~dB}$
Left eye
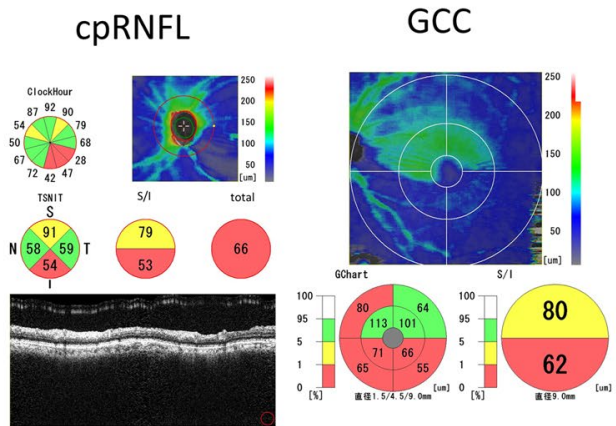

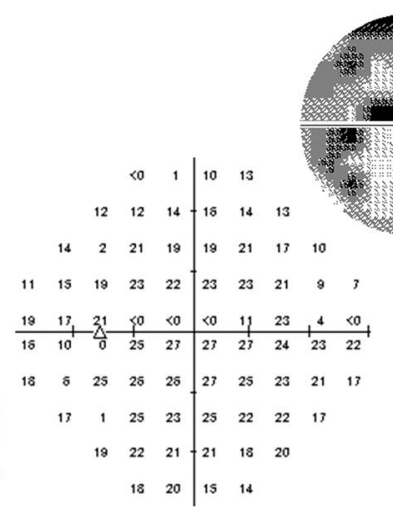

actual $\mathrm{MD}=-10.5 \mathrm{~dB}$ predicted MD: $-14.9 \mathrm{~dB}$ prediction error: $-4.4 \mathrm{~dB}$

FL: $4.8 \%$

FP: $0.0 \%$

FN: $13.0 \%$

MMSE total score: 16

Figure 1. A case example (86 year old male). MD values were predicted from OCT determined cpRNFL thickness and average GCC thickness through the leave-one-out cross-validation; the predicted MD value was calculated from the remaining 50 patients ( 92 eyes). Despite the reliable FL, FP and FN results, a markedly over(right eye) and under- (left eye) prediction was obtained. This patient had the lowest MMSE total score (16). MD: mean deviation, OCT: optical coherence tomography, cpRNFL: circumpapillary retinal nerve fiber layer, GCC: ganglion cell complex, FL: fixation loss, FP: false positive, FN: false negative, MMSE: Mini Mental State Examination.

\begin{tabular}{|l|l|}
\hline variables & number \\
\hline Gender (male:female) & $24: 27$ \\
\hline Age (years), mean \pm SD [range] & $80.8 \pm 3.8[76$ to 90$]$ \\
\hline Eye (right:left) & $47: 47$ \\
\hline MD $(\mathrm{dB})$, mean \pm SD [range] & $-6.5 \pm 4.7[-17.3$ to 0.9$]$ \\
\hline cpRNFL thickness $(\mu \mathrm{m})$ mean \pm sd [range] & $82.5 \pm 14.2[50.0$ to 113.0$]$ \\
\hline GCC thickness $(\mu \mathrm{m})$ mean \pm sd [range] & $77.7 \pm 12.0[50.5$ to 103.0$]$ \\
\hline FL $(\%)$ mean $\pm S D[$ range] & $9.7 \pm 9.4[0.0$ to 31.6$]$ \\
\hline FP $(\%)$ mean $\pm S D$ [range] & $2.1 \pm 3.1[0.0$ to 20.0$]$ \\
\hline FN $(\%)$ mean $\pm S D$ [range] & $5.3 \pm 6.5[0.0$ to 20.0$]$ \\
\hline total MMSE score, mean $\pm S D$ [range] & $23.3 \pm 3.2[16.0$ to 29.0$]$ \\
\hline
\end{tabular}

Table 1. Patient demographics. SD: standard deviation, MD: mean deviation, cpRNFL: circumpapillary retinal nerve fiber layer, GCC: ganglion cell complex, FL: fixation loss, FP: false positive, FN: false negative, MMSE: Mini Mental State Examination.

\section{Results}

Among the 104 eyes of 54 patients, 94 eyes of 51 patients had $\mathrm{FL}<33 \%, \mathrm{FP}<33 \%$ and $\mathrm{FN}<33 \%$. Table 1 lists the demographic data of patients: 58 eyes were phakic and 36 eyes were pseudophakic. Figure 2 shows a histogram of the MMSE total scores. Among the 51 patients, five patients (9\%) scored $28-30$ points (normal); 22 patients (41\%) scored $24-27$ points (MCI), and 24 patients (44\%) scored $\leq 23$ points (severe to moderate CI or almost diagnostic of dementia). No patients scored $<15$, so there was no severe CI among the patients in the current study. 


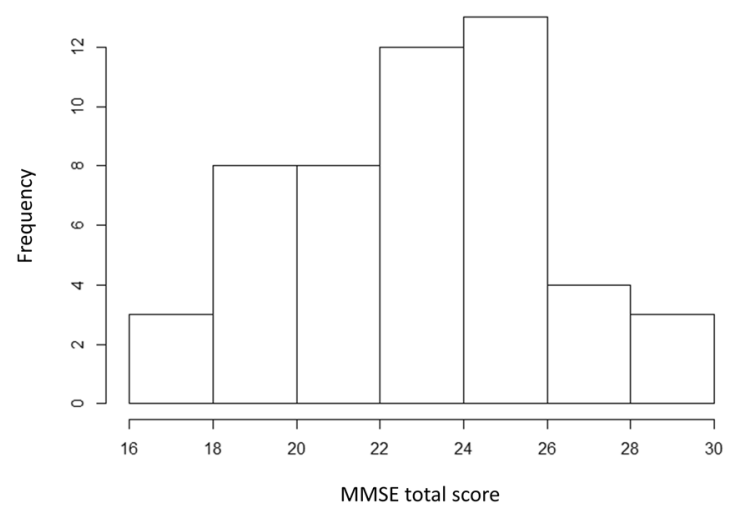

Figure 2. Histogram of Mini Mental State Examination (MMSE) total scores. The MMSE total score was $23.3 \pm 3.2$ (range, 16.0-29.0) (mean \pm standard deviation).

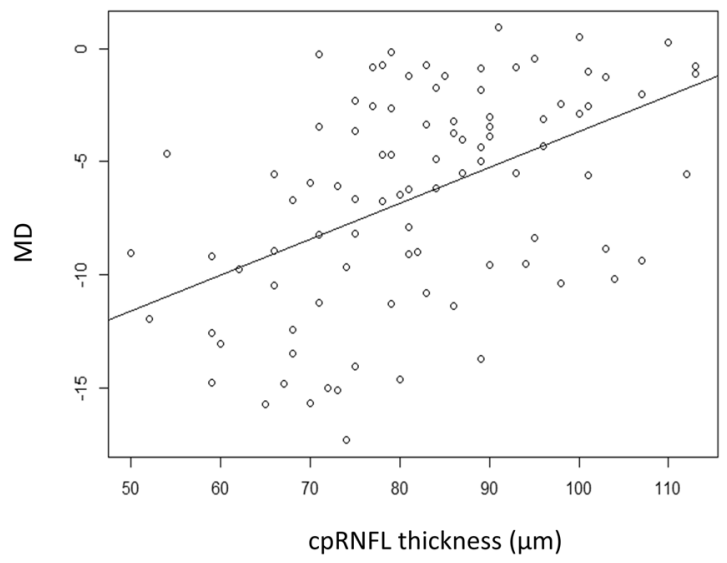

Figure 3. Relationship between the mean deviation (MD) and the circumpapillary retinal nerve fiber layer (cpRNFL) thickness. A significant relationship was observed between the MD and the cpRNFL thickness $[\mathrm{MD}=-19.6+0.16$ (standard error, 0.031; $\mathrm{p}<0.0001) \times \mathrm{cpRNFL}$ thickness (linear mixed model) $]$.

Figure 3 shows the relationship between cpRNFL thickness and the MD, which was significant $(\mathrm{p}<0.001$, linear mixed model). Similarly, a significant relationship was observed between GCC thickness and the MD $(\mathrm{p}<0.001$; Fig. 4). The MMSE total score was significantly related to age $(\mathrm{p}<0.001)$, but not to MD, FL, FP, or FN (Table 2); this analysis was carried out in 104 eyes of 54 patients including those with FL $>33 \%$, FP $>33 \%$ or FN $>33 \%$. As shown in Figs 5 and 6, the cpRNFL thickness and the GCC thickness did not significantly correlated with MMSE total score ( $\mathrm{p}=0.17$ and 0.23 , respectively, linear mixed model).

The multivariable linear mixed model between the MD and the cpRNFL thickness, GCC thickness, and age resulted in the following formula: $\mathrm{MD}=-26.3+0.064 \times$ cpRNFL thickness $+0.15 \times$ average GCC thickness $+0.034 \times$ age. Using the leave-one-out cross-validation, the MD was predicted using cpRNFL thickness, GCC thickness, and age, and the raw prediction error was $0.058 \pm 4.2(-9.7$ to 9.9$) \mathrm{dB}$. The optimal model prediction error was constructed by selecting optimal parameters from age, FL, FP, FN, and MMSE total score. MMSE total score, FL, FP, and FN were not selected, and only the intercept existed in the optimal model prediction error $_{\text {. }}$

The squared prediction error was calculated using the leave-one-out cross-validation method $[17.0 \pm 21.1$

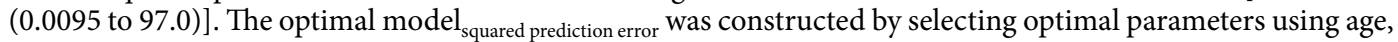

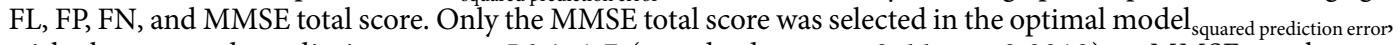
with the squared prediction error $=56.4-1.7$ (standard error $=0.66, \mathrm{p}=0.0012) \times$ MMSE total score $(\mathrm{AICc}=840.5)$. The relationship between squared prediction error and the MMSE total score is shown in Fig. 7. The optimal model ${ }_{\text {squared prediction error }}$ was significantly better than the model only with intercept (no independent variables included: AICc $=844.8): p=0.011$ (ANOVA test $)^{56}$. None of the models between squared prediction error and FL, FP, FN and age were significantly better than the model only with intercept ( $\mathrm{p}$ values between 0.35 and 0.95, ANOVA test), and adding any combinations of the four variables resulted in the decrease of AICc (AICc values were between 846.2 and 847.0).

\section{Discussion}

The present study measured the cognitive impairment in 94 eyes of 51 OAG patients $>75$ years of age, together with OCT and VF measurements. The cognitive impairment in elderly glaucoma patients and the relationship between cognitive decline and reliability measurements of the VF were assessed. The MD of the VF was predicted 


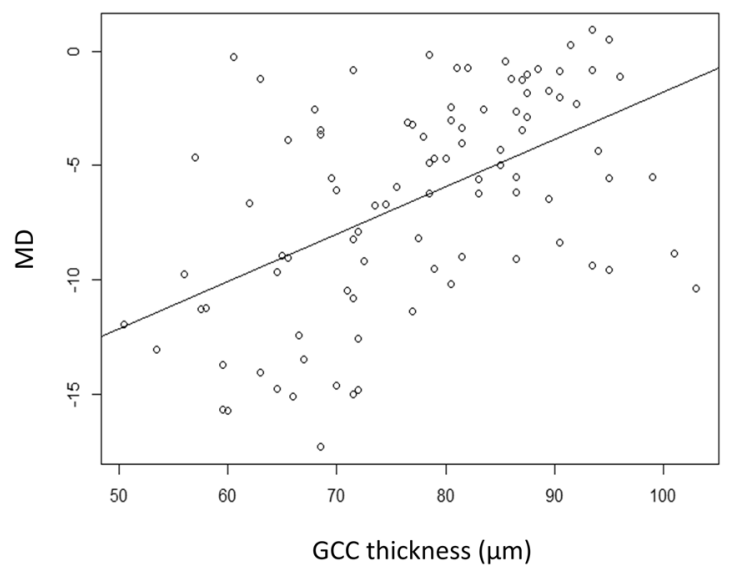

Figure 4. Relationship between the mean deviation (MD) and the ganglion cell complex (GCC) thickness. A significant relationship was observed between the MD and GCC thickness $[\mathrm{MD}=-22.5+0.21$ (standard error, 0.036; $\mathrm{p}<0.0001) \times$ GCC thickness (linear mixed model)].

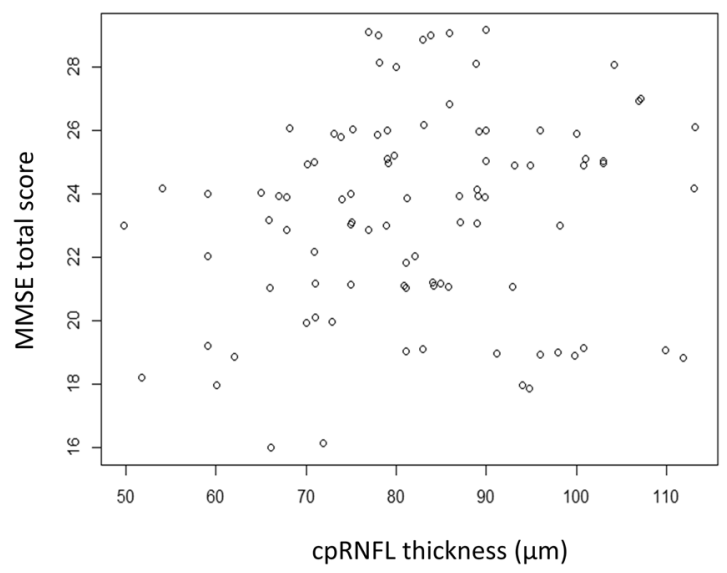

Figure 5. The relationship between the Mini Mental State Examination total score and the circumpapillary retinal nerve fiber layer thickness. No significant relationship was observed $(p=0.17$, linear mixed model).

\begin{tabular}{|l|l|l|l|l|}
\hline dependent variables & independent variable & coefficient & SE & p value \\
\hline MMSE total score & Age (years) & 0.13 & 0.031 & $<0.001$ \\
\hline MD (dB) & MMSE total score & 0.21 & 0.17 & 0.22 \\
\hline FL (\%) & MMSE total score & -0.059 & 0.35 & 0.87 \\
\hline FP (\%) & MMSE total score & 0.039 & 0.12 & 0.75 \\
\hline FN (\%) & MMSE total score & -0.31 & 0.24 & 0.21 \\
\hline
\end{tabular}

Table 2. The relationship between MMSE total score and age, MD, FL, FP, and FN. MMSE: Mini Mental State Examination, SD: standard deviation, MD: mean deviation, FL: fixation loss, FP: false positive, FN: false negative.

using OCT-measured cpRNFL and GCC thicknesses using the leave-one-out cross-validation method, and the relationship between the prediction error and the MMSE score was determined. The results revealed that the squared prediction error increased with decreasing MMSE score, indicating that prediction of the MD from OCT parameters was less accurate in these patients.

The onset of AD or dementia is sometimes difficult to diagnose, because it can be difficult to detect in its early stages. Furthermore, there is no effective treatment once the disease becomes clinically evident ${ }^{57}$. In a population-based study, $15-42 \%$ of the patients $\geq 65$ years of age were estimated to have $\mathrm{MCI}^{12}$, and the prevalence of cognitively impaired individuals $\geq 65$ years of age was estimated at $15 \%$ in Japan ${ }^{58}$. The results of the present study suggest that the prevalence of undiagnosed dementia or MCI is relatively high among elderly glaucoma patients. No patients were diagnosed with severe CI in the current study. However, of the 51 patients, 


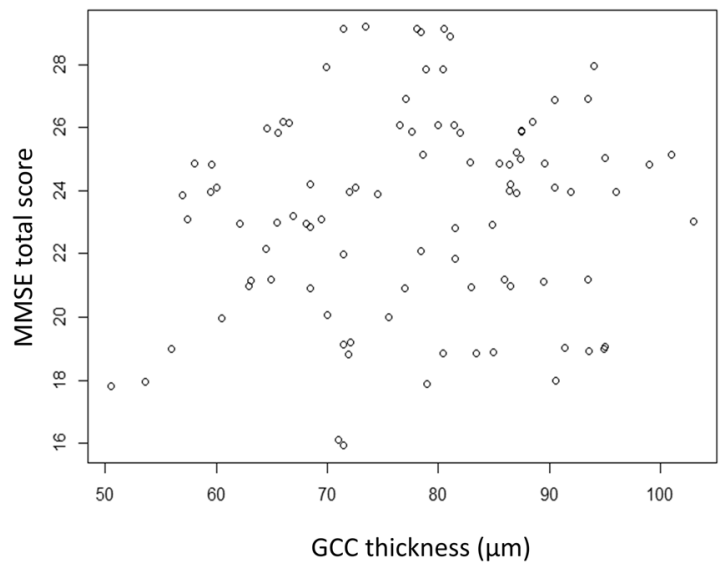

Figure 6. The relationship between the mean deviation and the ganglion cell complex thickness. No significant relationship was observed ( $\mathrm{p}=0.23$, linear mixed model).

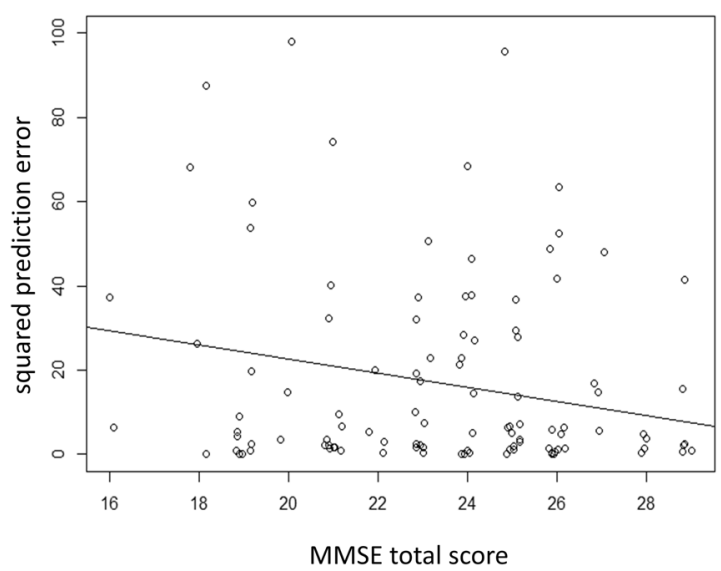

Figure 7. The relationship between the squared prediction error and the Mini Mental State Examination (MMSE) total score. Only the MMSE total score was selected in the optimal model squared prediction error $_{\text {The squared }}$ prediction error $=56.4-1.7($ standard error $=0.66, \mathrm{p}=0.0012) \times$ MMSE total score.

only five (9\%) had a normal MMSE score; 22 (41\%) were classified as MCI, and 24 (44\%) were classified as having moderate CI or suspected dementia (Fig. 2), although MMSE scores did not decrease with increased age $>75$ years (Table 2). It is important to note that none of the patients in the current study had been diagnosed with dementia; they lived their daily lives without any special assistance. Several previous studies have reported visual impairment-related cognitive hypofunction based on longitudinal research ${ }^{59,60}$. However, one recent study found no significant association between visual impairment and cognitive function in a 10-year follow-up study ${ }^{61}$. Further studies will therefore be needed to determine the association between glaucomatous visual impairment and cognitive function. In the Japanese governmental medical insurance system, individuals $\geq 75$ years of age are classified as 'latter-stage elderly', so increased attention should be addressed to these patients.

We have previously reported that the FL and FP were related to overestimation of the $\mathrm{VF}^{42}$; however this relationship was not observed in the current study. This may suggest that the usefulness of these reliability indices is limited in a population $\geq 75$ years of age. In contrast, the squared prediction error was related to MMSE total score (Fig. 7), and an increased squared prediction error was found among patients with low MMSE total

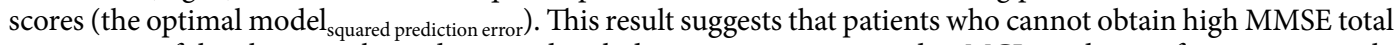
scores, even if they have not been diagnosed with dementia or categorized as MCI, tend to perform inaccurately with overly high or overly low VF measurements, suggesting that careful clinical consideration is needed when interpreting the VF results of patient populations with a high prevalence of MCI and early to moderate dementia. MMSE total score were selected as significant parameters in the model $_{\text {squared prediction error }}$, however not in the

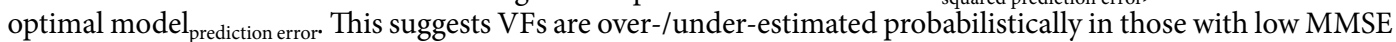
score. In a future study, the effects of MMSE total scores on the test-retest reproducibility of VF tests should be examined. As squaring the prediction error to calculate the squared prediction error changes the distribution and the value may not follow normal distribution. We used the linear mixed model which requires normal distribution, we log-transformed the squared prediction error. As a result, only the MMSE total score was included in the optimal model again (data not shown in Result). 
We recently reported that it is useful to include gaze tracking data, in addition to the FL, FP, and FN, to investigate test-retest reproducibility ${ }^{61}$ and over-and underestimation of the $\mathrm{VF}^{62,63}$. Recent studies have suggested that the FL, FP, and FN have limited usefulness in estimating the reliability of the VF ${ }^{16,20-22}$. Consistent with this possibility, in this study we observed no significant relationship between MMSE total score and any of the FL, FP, and FN values. Further studies should be performed to determine the usefulness of gaze tracking in the elderly population in assessing the reliability of the VF results, and the influence of cognitive impairment on gaze tracking.

In elderly patients, the VF with a narrower testing area, such as the 10-2 HFA VF, may be more accurately measured, because of various ocular conditions, including ptosis and miosis of the pupil. Also, the GCC scanning area corresponds mainly to the 10-2 VF. We recently reported that cpRNFL and GCC thicknesses were significantly correlated with HFA VF sensitivity in elderly glaucoma patients when reliable VF measurements were obtained $^{43}$. GCC thicknesses especially were strongly correlated with sensitivities in all test points within the central $5.8^{\circ}$ measured with the HFA 10-2 VF.

This study did not include use of the 10-2 HFA VF, which is a limitation that should be further investigated in future studies. Additionally, the MMSE consisted of 11 questions measures that test five areas of cognitive function: orientation, registration, attention and calculation, recall, and lauguage, but we did not investigate each of the scores, because of the small number of items (between 1-6). Several previous studies have reported that the thickness of the cpRNFL or GCC in subjects with AD and MCI is significantly thinner than in normal controls, so OCT could be a potentially useful diagnostic tool in the evaluation and follow-up of AD patients ${ }^{64,65}$. In the present study, there was no significant relationship between MMSE total score and the thicknesses of cpRNFL and GCC (Figs 4 and 5). The results of the present study therefore do not suggest that retinal nerve fiber degeneration caused by both glaucoma and central nervous system degeneration occur concurrently with thinning of the RNFL and GCC thicknesses measured by OCT. However, further studies with larger sample sizes and precise evaluations of cognitive functions including pathological examinations and neuroimaging will be needed to confirm the existence of a correlation between structural changes and cognitive impairment. Furthermore, care is required to optimize the value of OCT to follow-up elderly glaucoma patients.

In conclusion, a high prevalence of early to moderate cognitive impairment was observed in the elderly study population. Careful consideration is needed when interpreting the VF measurements of these patients, because the VF can be over- or underestimated, as suggested by the inconsistent structure-function relationships.

\section{References}

1. Quigley, H. A. \& Broman, A. T. The number of people with glaucoma worldwide in 2010 and 2020. Br J Ophthalmol. 90, 262-267 (2006).

2. Altangerel, U., Spaeth, G. L. \& Rhee, D. J. Visual function, disability, and psychological impact of glaucoma. Curr Opin Ophthalmol 14, $100-105(2003)$

3. Tielsch, J. M. et al. Racial variations in the prevalence of primary open-angle glaucoma. The Baltimore Eye Survey. JAMA 266, 369-374 (1991).

4. Mitchell, P., Smith, W., Attebo, K. \& Healey, P. R. Prevalence of open-angle glaucoma in Australia. The Blue Mountains Eye Study. Ophthalmology 103, 1661-1669 (1996).

5. Klein, B. E. et al. Prevalence of glaucoma. The Beaver Dam Eye Study. Ophthalmology. 99, 1499-1504 (1992).

6. Iwase, A. et al. The prevalence of primary open-angle glaucoma in Japanese: the Tajimi Study. Ophthalmology 111, 1641-1648 (2004).

7. Smits, C. H., Deeg, D. J., Kriegsman, D. M. \& Schmand, B. Cognitive functioning and health as determinants of mortality in an older population. Am J Epidemiol 150, 978-986 (1999).

8. Fratiglioni, L. et al. Incidence of dementia and major subtypes in Europe: a collaborative study of population-based cohorts. Neurologic Diseases in the Elderly Research Group. Neurology 54, S10-S15 (2000).

9. "Dementia Fact sheet No362". who.int. April 2012. Retrieved 28 November (2014).

10. Larson, E. B., Yaffe, K. \& Langa, K. M. New insights into the dementia epidemic. N Engl J Med 369, 2275-2277 (2013)

11. Petersen, R. C. et al. Mild cognitive impairment: clinical characterization and outcome. Arch Neurol 56, 303-330 (1999).

12. Petersen, R. C. et al. Mild cognitive impairment: a concept in evolution. J Intern Med 275, 214-228 (2014).

13. Jansonius, N. M. On the accuracy of measuring rates of visual field change in glaucoma. Br J Ophthalmol 94, 1404-1405 (2010).

14. Chauhan, B. C. et al. Practical recommendations for measuring rates of visual field change in glaucoma. Br J Ophthalmol 92, 569-573 (2008).

15. Vingrys, A. J. \& Demirel, S. The effect of fixational loss on perimetric thresholds and reliability. Perimetry Update 1992/93. Amsterdam: Kugler Publications (1992).

16. Demirel, S. \& Vingrys, A. J. Eye Movements During Perimetry and the Effect that Fixational Instability Has on Perimetric Outcomes. J Glaucoma 3, 28-35 (1994).

17. Fankhauser, F., Spahr, J. \& Bebie, H. Some aspects of the automation of perimetry. Surv Ophthalmol 22, 131-141 (1977).

18. Anderson, D. R. \& Patella, V. M. Automated Static Perimetry. 2nd ed. St. Louis: Mosby (1999).

19. Johnson, C. A., Sherman, K., Doyle, C. \& Wall, M. A comparison of false-negative responses for full threshold and SITA standard perimetry in glaucoma patients and normal observers. J Glaucoma 23, 288-292 (2014).

20. McMillan, T. A., Stewart, W. C. \& Hunt, H. H. Association of reliability with reproducibility of the glaucomatous visual field. Acta Ophthalmol (Copenh) 70, 665-670 (1992).

21. Katz, J. \& Sommer, A. Screening for glaucomatous visual field loss. The effect of patient reliability. Ophthalmology 97, 1032-1037 (1990).

22. Sanabria, O., Feuer, W. J. \& Anderson, D. R. Pseudo-loss of fixation in automated perimetry. Ophthalmology 98, 76-78 (1991).

23. Demirel, S. \& Vingrys, A. J. Fixational instability during perimetry and the blindspot monitor. Amsterdam: Perimetry Update 1992/1993. Kugler Publications (1992).

24. Bengtsson, B. \& Heiil, A. False-negative responses in glaucoma perimetry: indicators of patient performance or test reliability? Invest Ophthalmol Vis Sci 41, 2201-2204 (2000).

25. Chang, R. \& Budenz, D. L. New developments in optical coherence tomography for glaucoma. Curr Opin Ophthalmol 19, 127-135 (2008).

26. Huang, D. et al. Optical coherence tomography. Science 254, 1178-1181 (1991).

27. Cho, J. W. et al. Relationship between visual field sensitivity and macular ganglion cell complex thickness as measured by spectraldomain optical coherence tomography. Invest Ophthalmol Vis Sci 51, 6401-6407 (2010). 
28. Ueda, K. et al. Difference in correspondence between visual field defect and inner macular layer thickness measured using three types of spectral-domain OCT instruments. Jpn J Ophthalmol 59, 55-64 (2015).

29. Kim, N. R. et al. Structure-function relationship and diagnostic value of macular ganglion cell complex measurement using Fourierdomain OCT in glaucoma. Invest Ophthalmol Vis Sci 51, 4646-4651 (2010).

30. Rao, H. L. et al. Effect of spectrum bias on the diagnostic accuracy of spectral-domain optical coherence tomography in glaucoma. Invest Ophthalmol Vis Sci 53, 1058-1065 (2012).

31. Rao, H. L. et al. Retinal nerve fiber layer and macular inner retina measurements by spectral domain optical coherence tomograph in Indian eyes with early glaucoma. Eye (Lond) 26, 133-139 (2012).

32. Cordeiro, D. V. et al. Influence of optic disc size on the diagnostic performance of macular ganglion cell complex and peripapillary retinal nerve fiber layer analyses in glaucoma. Clin Ophthalmol 5, 1333-1337 (2011).

33. Shoji, T. et al. Assessment of glaucomatous changes in subjects with high myopia using spectral domain optical coherence tomography. Invest Ophthalmol Vis Sci 52, 1098-1102 (2011).

34. Tan, O. et al. Advanced Imaging for Glaucoma Study G. Mapping of macular substructures with optical coherence tomography for glaucoma diagnosis. Ophthalmology 115, 949-956 (2008).

35. Mwanza, J. C. et al. Macular ganglion cell-inner plexiform layer: automated detection and thickness reproducibility with spectral domain-optical coherence tomography in glaucoma. Invest Ophthalmol Vis Sci 52, 8323-8329 (2011).

36. Mori, S., Hangai, M., Sakamoto, A. \& Yoshimura, N. Spectral-domain optical coherence tomography measurement of macular volume for diagnosing glaucoma. J Glaucoma 19, 528-534 (2010).

37. Walji, M. F. et al. Detection and characterization of usability problems in structured data entry interfaces in dentistry. Int J Med Inform 82, 128-138 (2013).

38. Takagi, S. T., Kita, Y., Yagi, F. \& Tomita, G. Macular retinal ganglion cell complex damage in the apparently normal visual field of glaucomatous eyes with hemifield defects. J Glaucoma 218, 318-325 (2012).

39. Seong, M. et al. Macular and peripapillary retinal nerve fiber layer measurements by spectral domain optical coherence tomography in normal-tension glaucoma. Invest Ophthalmol Vis Sci 51, 1446-1452 (2010).

40. Mwanza, J. C. et al. Glaucoma diagnostic accuracy of ganglion cell-inner plexiform layer thickness: comparison with nerve fiber layer and optic nerve head. Ophthalmology 119, 1151-1158 (2012).

41. Kotowski, J. et al. Glaucoma discrimination of segmented cirrus spectral domain optical coherence tomography (SD-OCT) macular scans. Br J Ophthalmol 96, 1420-1425 (2012).

42. Ishiyama, Y., Murata, H., Hirasawa, H. \& Asaoka, R. Estimating the Usefulness of Humphrey Perimetry Gaze Tracking for Evaluating Structure-Function Relationship in Glaucoma. Invest Ophthalmol Vis Sci 56, 7801-7805 (2015).

43. Honjo, M. et al. Retinal Thickness and the Structure/Function Relationship in the Eyes of Older Adults with Glaucoma. PLoS One 10, e0141293 (2015).

44. Birt, C. M. et al. Analysis of reliability indices from Humphrey visual field tests in an urban glaucoma population. Ophthalmology 104, 1126-1130 (1997)

45. Petersen, R. C. et al. Practice parameter: early detection of dementia: mild cognitive impairment (an evidence-based review). Report of the Quality Standards Subcommittee of the American Academy of Neurology. Neurology 56, 1133-1142 (2001).

46. Matsuura, M., Hirasawa, K., Murata, H. \& Asaoka, R. The relationship between visual acuity and the reproducibility of visual field measurements in glaucoma patients. Invest Ophthalmol Vis Sci 56, 5630-5635 (2015).

47. Anderson, D. R. Automated Static Perimetry. 2nd ed. St. Louis: Mosby (1999).

48. Folstein, M. F., Folstein, S. E. \& McHugh, P. R. "Mini-mental state". A practical method for grading the cognitive state of patients for the clinician. J Psychiatr Res 12, 189-198 (1975).

49. Taniguchi, Y. et al. A prospective study of gait performance and subsequent cognitive decline in a general population of older Japanese. J Gerontol A Biol Sci Med Sci 67, 796-803 (2012).

50. Maki, N. et al. The validity of the MMSE and SMQ as screening tests for dementia in the elderly general population-a study of one rural community in Japan. Dement Geriatr Cogn Disord 11, 193-196 (2000).

51. Benson, A. D. et al. Screening for Early Alzheimer's Disease: Is There Still a Role for the Mini-Mental State Examination? Prim Care Companion J Clin Psychiatry 7, 62-69 (2005).

52. Mungas, D. In-office mental status testing: a practical guide. Geriatrics. 46, 54-58, 63, 66 (1991).

53. Morooka, S. et al. Wide 3-dimensional macular ganglion cell complex imaging with spectral-domain optical coherence tomography in glaucoma. Invest Ophthalmol Vis Sci 53, 4805-4812 (2012).

54. Nakanishi, H. et al. Sensitivity and specificity for detecting early glaucoma in eyes with high myopia from normative database of macular ganglion cell complex thickness obtained from normal non-myopic or highly myopic Asian eyes. Graefes Arch Clin Exp Ophthalmol 253, 1143-1152 (2015).

55. Yanagihara, H., Kamo, K., Imori, S. \& Satoh, K. Bias-corrected AIC for selecting variables in multinomial logistic regression models. Linear Algebra Appl 436, 4329-4341 (2012).

56. Wright, D. B. \& London, K. Modern Regression Techniques Using R. (SAGE Publications Ltd, 2009).

57. Salloway, S., Sperling, R. \& Brashear, H. R. Phase 3 trials of solanezumab and bapineuzumab for Alzheimer's disease. N Engl J Med 370, 1460 (2014).

58. Asada, T. Prevalence of dementia in Japan: past, present and future. Rinsho Shinkeigaku 52, 962-964 (2012).

59. Mine, M. et al. Association of Visual Acuity and Cognitive Impairment in Older Individuals: Fujiwara-kyo Eye Study. Biores Open Access 5, 228-234 (2016).

60. Rogers, M. A. \& Langa, K. M. Untreated poor vision: a contributing factor to late-life dementia. Am J Epidemiol 171, 728-735 (2010).

61. Hong, T. et al. Visual Impairment, Hearing Loss and Cognitive Function in an Older Population: Longitudinal Findings from the Blue Mountains Eye Study. PLoS One 11, e0147646 (2016).

62. Ishiyama, Y., Murata, H., Mayama, C. \& Asaoka, R. An objective evaluation of gaze tracking in Humphrey Perimetry and the relation with the reproducibility of visual fields -a pilot study in glaucoma. Invest Ophthalmol Vis Sci 55, 8149-8152 (2014).

63. Ishiyama, Y., Murata, H. \& Asaoka, R. The Usefulness of Gaze Tracking as an Index of Visual Field Reliability in Glaucoma Patients. Invest Ophthalmol Vis Sci 56, 6233-6236 (2015).

64. Kelsey, L. T. et al. A systematic review and meta-analysis of retinal nerve fiber layer change in dementia, using optical coherence tomography. Alzheimers Dement (Amst) 1, 136-143 (2015)

65. Cunha, L. P. et al. Macular Thickness Measurements with Frequency Domain-OCT for Quantification of Retinal Neural Loss and its Correlation with Cognitive Impairment in Alzheimer’s Disease. PLoS One 11, e0153830 (2016).

\section{Acknowledgements}

We are grateful to Yuko Sodeyama for her help with the cognitive function test. The statistical analysis in this document has been consulted with a statistician and proofread, and the English in this document has been checked by at least two professional editors, both native speakers of English. For a certificate, please see: http:// www.textcheck.com/certificate/FQHeQu. Japan Society for the Promotion of Science 15K10854 to M.H. Japan Society for the Promotion of Science 17K11418 to R.A. 


\section{Author Contributions}

M.H. and R.A. wrote the main manuscript text and J.N. and T.H. prepared Figs 1-2. All authors reviewed the manuscript.

\section{Additional Information}

Competing Interests: The authors declare that they have no competing interests.

Publisher's note: Springer Nature remains neutral with regard to jurisdictional claims in published maps and institutional affiliations.

(c) (i) Open Access This article is licensed under a Creative Commons Attribution 4.0 International License, which permits use, sharing, adaptation, distribution and reproduction in any medium or format, as long as you give appropriate credit to the original author(s) and the source, provide a link to the Creative Commons license, and indicate if changes were made. The images or other third party material in this article are included in the article's Creative Commons license, unless indicated otherwise in a credit line to the material. If material is not included in the article's Creative Commons license and your intended use is not permitted by statutory regulation or exceeds the permitted use, you will need to obtain permission directly from the copyright holder. To view a copy of this license, visit http://creativecommons.org/licenses/by/4.0/.

(c) The Author(s) 2017 